New insights into the melanophilin (MLPH) gene controlling coat color phenotypes in American mink

Cirera Salicio, Susanna; Markakis, Marios N.; Christensen, Knud; Anistoroaei, Razvan Marian

Published in:

Gene

DOI:

10.1016/j.gene.2013.05.047

Publication date:

2013

Document version

Early version, also known as pre-print

Citation for published version (APA):

Cirera Salicio, S., Markakis, M. N., Christensen, K., \& Anistoroaei, R. M. (2013). New insights into the melanophilin (MLPH) gene controlling coat color phenotypes in American mink. Gene, 527(1), 48-54. https://doi.org/10.1016/j.gene.2013.05.047 


\title{
New insights into the melanophilin $(M L P H)$ gene controlling coat color phenotypes in American mink
}

\author{
Susanna Cirera ${ }^{a}$, Marios N. Markakis ${ }^{\mathrm{b}}$, Knud Christensen ${ }^{\mathrm{a}}$, Razvan Anistoroaei a,* \\ a University of Copenhagen, Faculty of Health and Medical Science, Department of Veterinary Clinical and Animal Sciences, Division of Genetics, Bioinformatics and Breeding, \\ 1870 Frederiksberg C, Denmark \\ ${ }^{\mathrm{b}}$ University of Antwerp, Department of Biology, Plant Growth and Development, Groenenborgerlaan 171, 2020 Antwerpen, Belgium
}

\section{A R T I C L E I N F O}

\section{Article history:}

Accepted 23 May 2013

Available online 6 June 2013

\section{Keywords}

American mink

Neovison vison

Silverblue

Violet

$\mathrm{MLPH}$

Deletion

\begin{abstract}
A B S T R A C T
The mutation causing the Silverblue color type (pp) is one of the most used recessive mutations within American mink (Neovison vison) fur farming, since it is involved in some of the popular color types such as Violet and Saphire which originate from a combination of recessive mutations. In the present study, the genomic and mRNA sequences of the melanophilin (MLPH) gene were studied in Violet, Silverblue and wild-type $(w t)$ mink animals. Although breeding schemes and previous literature indicates that the Violet (aammpp) phenotype is a triple recessive color type involving the same locus as the Silverblue ( $p p$ ) color type, our findings indicate different genotypes at the MLPH locus. Upon comparison at genomic level, we identified two deletions of the entire intron 7 and of the $5^{\prime}$ end of intron 8 in the sequence of the Silverblue MLPH gene. When investigating the mRNA, the Silverblue animals completely lack exon 8, which encodes 65 residues, of which 47 define the Myosin Va (MYO5A) binding domain. This may cause the incorrect anchoring of the MLPH protein to MYO5A in Silverblue animals, resulting in an improper pigmentation as seen in diluted phenotypes. Additionally, in the MLPH mRNA of wt, Violet and Silverblue phenotypes, part of intron 8 is retained resulting in a truncated MLPH protein, which is 359 residues long in wt and Violet and 284 residues long in Silverblue. Subsequently, our findings point out that the missing actin-binding domain, in neither of the 3 analyzed phenotypes affects the transport of melanosomes or the consequent final pigmentation. Moreover, the loss of the major part of the MYO5A domain in the Silverblue MLPH protein seems to be the responsible for the dilute phenotype. Based on our genomic DNA data, genetic tests for selecting Silverblue and Violet carrier animals can be performed in American mink.
\end{abstract}

(c) 2013 Elsevier B.V. All rights reserved.

\section{Introduction}

The mutation causing the Silverblue color type is one of the most used recessive mutations within mink fur farming. It is used in homozygous form, such as in pure Silverblue $(p p)$ or is part of some of the popular color determining genotypes in which several recessive mutations are combined, such as for instance in the light sand color type Pearl (ppmm), Violet (aammpp) and the Bluish Sapphire (aapp). In the latter two phenotypes, also the LYST gene is involved

Abbreviations: MLPH, melanophilin; $p p$, Silverblue genotype; wt, wild-type; aammpp, Violet genotype; MYO5A, Myosin Va; mRNA, messenger RNA; ppmm, Pearl genotype; aapp, Bluish Sapphire genotype; LYST, lysosomal trafficking regulator gene; $p^{s} p^{s}$, Steelblue genotype; RAB27A, member RAS oncogene family; SNP, single nucleotide polymorphism; PCR, Polymerase Chain Reaction; ng, nanograms; BLAST, Basic Local Alignment Search Tool; cDNA, Complementary DNA; mg, milligrams; Sdw, sterile distillated water; BAC, Bacterial artificial chromosome; UTR, untranslated region; nt, nucleotides; bp, base pair; LOD, logarithm (base 10) of odds; G, Glutamine; H, Histidine; S, Serine; N, Asparagine; ORF, open reading frame; NCBI, National Center for Biotechnology Information; DeltaAB, Slac2-a deletion mutant; KA, Slac2-a point mutant.

* Corresponding author. Tel.: + 4535333050.

E-mail address: ran@sund.ku.dk (R. Anistoroaei).
(Anistoroaei et al., 2013). The Silverblue phenotype on a wild type mink background is characterized by a bluish gray color, with slightly darker tail and feet in comparison to the rest of the body (Fig. 1A). The nose and eyes are lighter in color than the wild-type (wt) (Fig. 1C). Violet (also known as Moyle Sapphire) is a pale blue type with red eyes (Fig. 1B) and is less brownish in comparison to the Silverblue phenotype.

As previously reported, the Silverblue mink coat color is inherited as a Mendelian autosomal recessive trait and has been determined to involve the MLPH gene (Anistoroaei and Christensen, 2007). In the mink genetics literature (Clausen et al., 2012) two alleles are described at the Silver locus; Silverblue $(p p)$ and Steelblue $\left(p^{s} p^{s}\right)$. Important to mention is the fact that until now, none of the classical $a$ to $e$ color genes have been identified in the American mink; therefore the mink genetics have developed an independent nomenclature (Clausen et al., 2012).

The dilution phenotype for coat color occurs in many mammalian species and is characterized by a defective transport of melanosomes to the melanocytes, leading to large clumps of pigments around the perinuclear region and resulting in a lighter coat color of the animal 

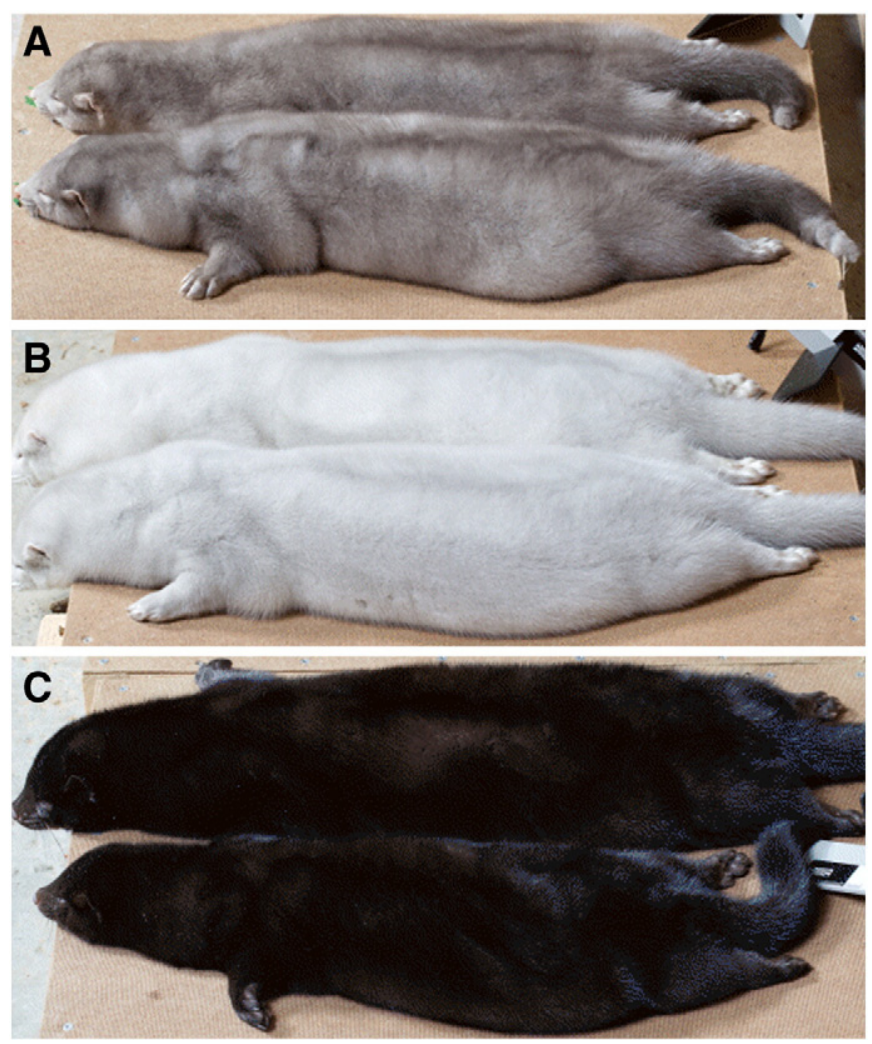

Fig. 1. A. Silverblue mink; B Violet mink; C wt mink.

(Barral and Seabra, 2004). The molecular biology behind pigmentation is rather complex, involving many genes (Passeron et al., 2005). Mutations in three different genes i.e. Myosin Va (MYO5A), RAB27A and $M L P H$, have been described to be responsible for the diluted phenotype (Hume et al., 2007). The products of these three genes collaborate to anchor the melanosomes to the actin cytoskeleton (Nascimento et al., 2003), forming a protein-actin complex which directs the transport of the melanosomes within the cell. In mouse, three coat color mutants: dilute, ashen and leaden have been characterized, in which the abovementioned three genes are involved (Matesic et al., 2001; Mercer et al., 1991; Wilson et al., 2000). In humans, mutations in the MLPH gene have been shown to cause the Griscelli syndrome type III, an autosomal recessive disorder characterized by pigment dilution of the skin and hair (OMIM 609227) (Menasche et al., 2003). In cats, a single base pair deletion in exon 2 of the MLPH gene causes the feline dilute coat color phenotype (Ishida et al., 2006), while in dogs, a SNP at the splice donor site of exon 1 of the MLPH gene has been described as a candidate causal mutation for coat color dilution (Drögemüller et al., 2007). Similarly, in poultry, Bed'hom et al. (2008) found a large deletion in the MLPH gene region in lavender quails. More recently, Vaez et al. (2008) have reported a causative non-synonymous single nucleotide polymorphism (SNP) (R35W) in the MLPH gene, on the basis of the lavender phenotype in chickens.

The MLPH gene is relatively well conserved between species. A MLPH protein consists of three domains of which two are very important: the RAB27 similar domain, which attaches the MLPH to the RAB27 protein at the surface of the melanosome, and the Myosin Va (MYO5A) domain, which binds specifically the MLPH to the Myosin. In human and mice, it is postulated that the dilution phenotypes are caused by the inability of MLPH to bind RAB27 and link it to MYO5A. As the MLPH sequence in $w t$ mink has been generated (Anistoroaei et al., 2011; Acc: JF288169) we were able to sequence and compare the region between the Silverblue, the Violet and the wt phenotypes in mink at genomic- and mRNA levels. We discuss the functional implications of the different mutations found in the light of coat color phenotypes in American mink. Furthermore, genetic tests are proposed to select for carriers of Silverblue and Violet phenotypes in mink.

\section{Material and methods}

\subsection{Pedigree and genotyping}

A half-sib family originating from a Danish farm in Sealand, Denmark, with 22 offspring that segregated for the Silverblue color described in Anistoroaei and Christensen (2007; Supplementary Fig. 1A) was genotyped with three new microsatellite markers: RAN79, RAN80 and RAN81 (Table 1). These markers were developed from the assembled contig (\#JF288169) containing the MLPH gene (Anistoroaei et al., 2011). Additionally, a half-sib family originating from the Taastrup Experimental farm of the Faculty of Life Sciences, University of Copenhagen, Denmark, with 22 offspring that segregated for the Violet color was genotyped with the same markers. Briefly, genomic DNA was extracted from tongues of the euthanized mink by using a Promega DNA extraction kit (\#A1125). DNA amplification was performed with fluorescently labeled forward primers (HEX and NED) on an ABI Prism 3130 sequencer, and genotypes were analyzed using Genscan analysis (V.3.1.2) software (Applied Biosystems). The dilute animals were classified as homozygous for the recessive mutated dilute allele ( $p p)$ and the other types of colored mink were classified as carrying at least a single copy of the dominant wild-type allele $(P)$.

\subsection{Sequence analysis of the MLPH gene}

The genomic contig containing the MLPH gene (\#JF288169) was analyzed in silico in order to predict the exonic sequences of the $M L P H$ gene with human, mouse, dog and cat sequences as references. Furthermore, transcriptome data from a wt mink (Anistoroaei et al., 2012a) was used to identify the possible transcripts of the $M L P H$ gene. The analyses were performed by using Spidey software (http:// www.ncbi.nlm.nih.gov/spidey/).

Primers were designed from the flanking intronic regions of all predicted exons of the MLPH gene of mink (\#JF288169; Anistoroaei et al., 2011) using Primer3 software (http://frodo.wi.mit.edu/cgibin/primer3/primer3_www.cgi). As the genetic material from the Violet family was freshly prepared, we started our sequencing analysis based on Violet instead of pure Silverblue. A total of 15 primer pairs were designed and used to amplify each of the 16 exons and an upstream region of the MLPH gene in wt and Violet (see Table 2 for primer sequences). PCRs were carried out at standard conditions at $60{ }^{\circ} \mathrm{C}$ annealing and $2 \mathrm{mM}$ magnesium. In order to verify the SNPs found in exon 8 and intron 8, also 5 Violet and 2 Silverblue animals from the original study (Anistoroaei and Christensen, 2007) as well as $2 w t$ animals were sequenced for this region. PCR products were cleaned using a PCR purification kit (Qiagen, Germany) and quantified by NanoDrop ND-1000 spectrophotometer (Thermoscientific, USA). Subsequently, $40-80$ ng of DNA was used for each sequencing reaction using BigDye terminators (Applied Biosystems) following the

Table 1

Microsatellite markers used for genotyping the Silverblue and Violet mink families.

\begin{tabular}{llll}
\hline Primer pair name & Forward and reverse primers $\left(5^{\prime} 3^{\prime}\right)$ & $\begin{array}{l}\text { Repeat } \\
\text { motif }\end{array}$ & Alleles size \\
\hline RAN79 & $\begin{array}{l}\text { F: CCTGCAGACAGTGAGAGCTG } \\
\text { R: CAACCCCCTTGGGAAAATAC }\end{array}$ & $(\mathrm{GT})_{22}$ & $200-210$ \\
RAN80 & $\begin{array}{l}\text { F: CCAGCCAAGGATCTAAGCAC } \\
\text { R: GTCGGGCTCCCTTTATTCTC }\end{array}$ & $(\mathrm{CT})_{12}$ & $235-241$ \\
RAN81 & F: GGGATTCTCTCTCCCTCACC & $(\mathrm{TC})_{13}$ & $192-205$ \\
& R: GGCTACATTCAAGGGACACA & & \\
\hline
\end{tabular}


manufacturer's instructions. The sequencing reactions were cleaned by a Millipore system and sequenced using a 3130XL sequencing machine (Applied Biosystems). DNA sequences were processed by means of Lasergene (DNA Star Inc.), and they were compared using BLAST bl2seq.

\subsection{RNA isolation, $c D N A$ synthesis and $c D N A$ sequencing}

Four Silverblue and four wt mink puppies were euthanized and several tissues were collected including skin, lung and kidney and placed at $-80{ }^{\circ} \mathrm{C}$ until use. For cDNA preparation, around $100 \mathrm{mg}$ of tissue was used for total RNA isolation using the TRI Reagent ${ }^{\circledR}$ protocol according to the manufacturer's recommendations. Homogenization was performed using a gentleMACS ${ }^{\mathrm{TM}}$ Octo Dissociator machine (Milteny Biotec, GmbH, Germany). Remaining genomic DNA was degraded using the RNase-free DNAse step from the RNeasy micro kit (Qiagen, Germany). Quantity and quality of the isolated RNA was measured in a NanoDrop ND-1000 (Thermoscientific, USA) and visually inspected after running it in a 1.4\% agarose gel. All RNA samples were kept at -80 until cDNA synthesis. Briefly, one $\mu \mathrm{g}$ of total RNA from each tissue and animal was reverse transcribed at $42{ }^{\circ} \mathrm{C}$ using Improm-II ${ }^{\mathrm{TM}}$ reverse transcriptase (Promega) and a mixture 3:1 of random hexamers: oligodT, according to the manufacturer's recommendations. The four cDNA samples for each phenotype and tissue were pooled and diluted 1:8 in RNAse-free Sdw prior to performing

Table 2

Primer sequences and ampliqon size for all MLPH exons.

\begin{tabular}{|c|c|c|}
\hline Gene name & Primer sequences & Ampliqon size \\
\hline UpMLPH & $\begin{array}{l}\text { fw: 5' } 5^{\prime} \text { TCACTCTCTTCTGGCTTGACTT 3' } \\
\text { rev: 5' CATTTTCCAAACAGAGCTCT 3' }\end{array}$ & 563 bp \\
\hline MLPH.Exon 1 & $\begin{array}{l}\text { fw: 5' TGTTCAAAAAGCTCTAAAAGCCTCC 3' } \\
\text { rev: 5' GGTATTATAGAGGGCACAGCTTGCA 3' }\end{array}$ & $492 \mathrm{pb}$ \\
\hline MLPH.Exon2 & $\begin{array}{l}\text { fw: 5' TTAGAAAAGCATTACACCCAGGTCC 3' } \\
\text { rev: 5' GCATTGATGCTTCCTGCTGTCTG 3' }\end{array}$ & 368 bp \\
\hline MLPH.Exon3-4 & $\begin{array}{l}\text { fw: 5' TGCCTGGGGGTGACTTACCTGT 3' } \\
\text { rev: 5' TGGGAACAAAGAGCGAGGCC 3' }\end{array}$ & 694 bp \\
\hline MLPH.Exon5 & $\begin{array}{l}\text { fw: 5' CTCGCCTGACATCCACAGTA 3' } \\
\text { rev: 5' TCGCCGACTCCTTACATCTC 3' }\end{array}$ & 356 bp \\
\hline MLPH.Exon6 & $\begin{array}{l}\text { fw: 5' CAATCTCTCTCCCTGGGTTG 3' } \\
\text { rev: 5' GACCGGGGAAGACTTACTTTG 5' }\end{array}$ & 164 bp \\
\hline MLPH.Exon7 & $\begin{array}{l}\text { fw: 5' GCCCCTTAGTGCTGCCTTGA 3' } \\
\text { rev: 5' TGGCCGTCAAACTCCTTCCC 3' }\end{array}$ & 380 bp \\
\hline MLPH.Exon8 & $\begin{array}{l}\text { fw: } 5^{\prime} \text { TCCACGGACACATGATACAGAGTG 3' } \\
\text { rev: 5' ACCCCTTCCCCAATGCAGAG 3' }\end{array}$ & 433 bp \\
\hline MLPH.Exon9 & $\begin{array}{l}\text { fw: 5' GTGCACTGGCTTCCAACTTT 3' } \\
\text { rev: 5' AAAGCATCAGCAGGAAGGAA 3' }\end{array}$ & 242 bp \\
\hline MLPH.Exon 10 & $\begin{array}{l}\text { fw: } 5^{\prime} \text { CACACAGAGGACCAGCTGAA 3' } \\
\text { rev: 5' AGGCAGCCTCACCTCCAG 3' }\end{array}$ & 242 bp \\
\hline MLPH.Exon11 & $\begin{array}{l}\text { fw: 5' GGCCCCAACAAGAGCTGCAT 3' } \\
\text { rev: 5' AGCTTTGGTGCACTGTCCACAG 3' }\end{array}$ & 396 bp \\
\hline MLPH.Exon12 & $\begin{array}{l}\text { fw: 5' CTGCATGGGGGTGATCCTTGA 3' } \\
\text { rev: 5' TGTTCCAGTCACACCCAGCCAG 3' }\end{array}$ & 432 bp \\
\hline MLPH.Exon13 & $\begin{array}{l}\text { fw: 5' AAACAAGCCACCGTCAGGTA 3' } \\
\text { rev: 5' GCTGAGCTGCAAGGTGTGT 3' }\end{array}$ & $373 \mathrm{bp}$ \\
\hline MLPH.Exon 14 & $\begin{array}{l}\text { fw: 5' TGGTGTTTCATTTATCGATACCGTG 3' } \\
\text { rev: 5' CAGTGCAGAGAATTCCAGAACACC 3' }\end{array}$ & 444 bp \\
\hline MLPH.Exon 15 & $\begin{array}{l}\text { fw: 5' AGACGGGACCTCCTTTCATC 3' } \\
\text { rev: 5' TCCCATTTTCTCTGCTCTGG 3' }\end{array}$ & 196 bp \\
\hline MLPH.Exon16 & $\begin{array}{l}\text { fw: 5' TGGTGTTTGTGTTCAAATGAAACGG 3' } \\
\text { rev: 5' GCCAAAAAAGAAACTGATCCCCTTT 3' }\end{array}$ & 390 bp \\
\hline Exon7. $M L P H^{\mathrm{a}}$ & Fw: 5' TTTGAGGCTGACTCTGACGA 3' & \\
\hline Intron7. $M L P H^{\mathrm{a}}$ & fw: 5' TCCACGGACACATGATACAGAGTG 3' & \\
\hline Exon8. $M L P H^{\mathrm{a}}$ & fw: 5' GTGTGCTCAAACCTTCATGG 3' & \\
\hline Exon8. $M L P H^{\mathrm{a}}$ & rev: 5' CAGGGCTGTCCTGCGGGACT 3' & \\
\hline Intron8. 1.MLPH ${ }^{\mathrm{a}}$ & rev: 5' ACCCCTTCCCCAATGCAGAG 3' & \\
\hline Intron8. 2.MLPH ${ }^{\mathrm{a}}$ & rev: 5' CTGAACTCCTGAACCCACAC 3' & \\
\hline Exon9.1. $M L P H^{\mathrm{a}}$ & rev: 5' GCACTGAACAGGCCACAGTA 3' & \\
\hline Exon9.2. $M L P H^{\mathrm{a}}$ & rev: 5' TGGTGGAGTATTTGCTCCTCTT 3' & \\
\hline
\end{tabular}

\footnotetext{
a Extra primers sequences designed for the region from exon 7 to exon 9
}

the PCR. Additionally, available cDNA from lung of a Violet animal was included in the study.

Primer sequences for the region encompassed by exon 7 to exon 9 were designed using Primer3 software (http://frodo.wi.mit.edu; Rozen and Skaletsky, 2000) (Table 2) and used in different combinations. The PCR products resulting from amplification of kidney and lung cDNAs (from both wt and Silverblue) contained more DNA and were selected for sequencing. PCR products were cleaned and sequenced as described above.

\section{Results}

\subsection{The mink MLPH gene as derived from the BAC contig}

Based on the analysis of the assembled genomic contig containing the MLPH gene (\#JF288169), derived from the CHORI-231 wt color American mink BAC library (Anistoroaei et al., 2011), the mink MLPH gene (UTRs and coding regions) is predicted to be represented by 16 exons and 2450 nucleotides (nt). Based on in silico comparisons within mink, human, mouse, cat and dog sequences, the coding region of the MLPH gene virtually consists of $1719 \mathrm{bp}$, which theoretically would translate into 573 amino acids. However, the results of the present study (see description below) indicate that the MLPH transcript in wt mink is only $1077 \mathrm{bp}$ long, yielding a protein of 359 amino acid residues (Fig. 2).

\subsection{Genotyping of the mink families}

The genomic contig of the MLPH gene (\#JF288169) provided $21 \mathrm{di}-$, tetra-, penta- and hexa-nucleotide repeated motifs, which could subsequently be developed into microsatellite markers. Microsatellite markers RAN79, RAN80 and RAN81 were further developed and characterized to be used in this study.

The new markers fully confirmed the association to the Silverblue phenotype. In addition, in another study, RAN 79 was linked to chromosome 3 and in situ hybridized to American mink 3q1.3-2.2 (Anistoroaei et al., 2012b). The genotyping of the 3 above mentioned markers for animals within the family segregating for the Silverblue phenotype (Supplementary Fig. 1) revealed informative polymorphisms for all of them, each of them yielding a LOD score value of 4.52 and perfect co-segregation with the Silverblue phenotype. The markers were not informative for the Violet family.

\subsection{Analysis of the genomic sequence of the MLPH gene in Silverblue, Violet and $w t$}

Sequencing the region encompassed from exon 7 to exon 9 in the gDNA of 2 Silverblue animals revealed the complete loss of intron 7 and the first part of intron 8 (from position 1 to position 2224). The same genomic regions in $w t$ and Violet revealed no insertion/deletion and only three SNPs: 1 . a non-synonymous SNP segregating in both the Violet and $w t$ phenotypes is located in exon 8 (c-785G $>C$ ), which results in an amino-acid variation in the MLPH protein (from Glutamine $(G)$, an uncharged polar residue, to Histidine $(H)$, a basic residue); 2. a synonymous SNP in exon 8 (c-885G $>$ A) where both Violet and $w t$ are heterozygous and 3. one SNP at the acceptor site of exon8/intron8, where the $G$ nucleotide at the splice site of the $w t$ is mutated to an A in the Violet (c.901+1G $>$ A) (Fig. 4).

The rest of the exons and $5^{\prime}$ UTR region of the MLPH gene were sequenced in Violet and $w t$. We identified three SNPs and one insertion: two of the SNPs occur in a region located approximately 5500 nucleotides upstream of the MLPH gene ( $c-5706 \mathrm{~T}>\mathrm{C}$ and $\mathrm{c}-5703 \mathrm{~A}>\mathrm{G}$ ) where we also found a 5 nucleotides insertion in Violet (a 5847CATTT sequence in wt compared to 5847GGGGTAGGAA in Violet); the third SNP segregates in both the Violet and $w t$ phenotypes at position 10 of exon 2 (c-11T $>$ G) (see Fig. 4). 


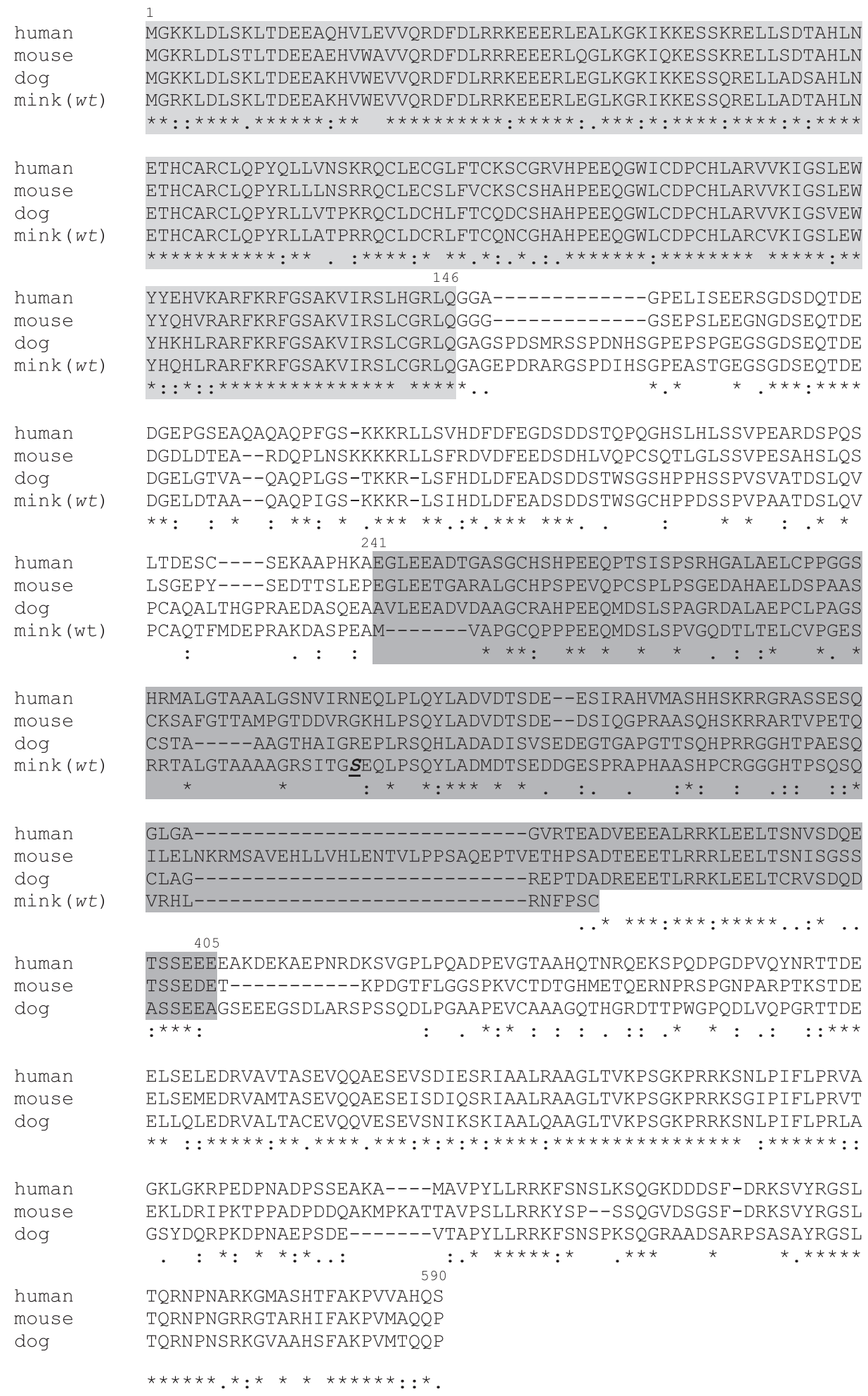

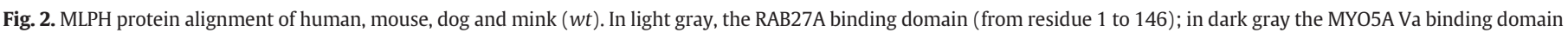

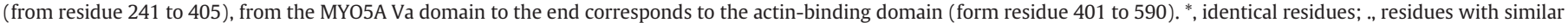

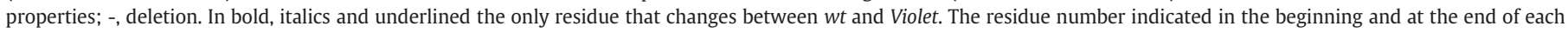
domain is based on the mouse sequence which is 590 amino acids long.

\subsection{Analysis of the MRNA and protein sequences of the MLPH gene in mink}

In order to investigate the effect of the SNP affecting the $5^{\prime}$ splice site in intron 8, we decided to sequence the cDNA from wt (lung and kidney), Violet (only lung available) and Silverblue (lung and kidney) animals. Different primer combinations were used in order to cover the region encompassed from the end of exon 7 to the beginning of exon 9 (see Table 2 for primer sequences).

The sequencing revealed that the MLPH transcripts from $w t$, Violet and Silverblue had retained part of intron 8 (see the light gray box in Fig. 4). In fact, the sequence from position 1 to position 2224 in intron 
8 is spliced out in wt and Violet and the rest of the intron (581 bp) is retained in the cDNA. This retained sequence gives rise to a truncated protein in $w t$ and Violet due to the introduction of a stop codon after position 177 of the intron, resulting in a MLPH protein 359 residues long (Fig. 2). In this retained intron we found 1 non-synonymous SNP between $w t$ and Violet in position 19 of the intron 8 which results in a change from a Serine (S, neutral polar amino acid) in $w t$ to an Asparagine (N, neutral non-polar amino acid) in Violet. In Silverblue the situation is even more complex as, surprisingly, the mRNA sequence lacks the entire exon 8 , leading to a shift in the open reading frame (ORF). Therefore, the retained sequence of part of intron 8 introduces a stop codon at nt 147 resulting in a hypothetical protein consisting of 284 residues, where the last 49 residues are totally different from the $w t$ and Violet phenotypes (Fig. 3).

When using the sequence of this partially retained intron to BLAST against the non-redundant database at National Center for Biotechnology Information (NCBI), we found homology to a MLPH transcript variant in ferret (Mustela putorius furo) and Giant panda (Ailuropoda melanoleuca).

When examining the different domains coded in the MLPH protein (Philipp et al., 2005), the truncated protein in wt and Violet mink results only in the lack of the last 28 residues of the MYO5A binding domain and the complete loss of the actin binding site. In Silverblue, the MLPH protein lacks the majority of the MYO5A Va binding domain and the complete actin binding domain. Additionally, the last 49 residues of the protein are very different between the $w t /$ Violet mink (Fig. 3) and the other studied mammals.

\subsection{Genetic test}

The fixed polymorphisms between the Violet and $w t$ found in the $M L P H$ gene region (Fig. 4) can be readily used for genetic testing. For the SNPs, TaqMan assays (developed by Applied Biosystems) can be designed for the 5 bp insertion in Violet and the two deletions found in Silverblue, acrylamide and agarose electrophoresis systems can be applied respectively.

\section{Discussion}

In our previous study (Anistoroaei and Christensen, 2007) we mapped the MLPH gene, which is involved in the Silverblue phenotype in mink. The phenotype is similar to the dilution color in other species such as dog, cat, and chicken. As the resources available for the American mink (BAC library) allowed more investigations, we continued our study in order to clearly confirm and characterize the role of the MLPH gene in the expression of this phenotype. We started by re-genotyping the Silverblue family material from Anistoroaei and Christensen (2007) with newly developed microsatellite markers (RAN79, RAN80 and RAN81; (Table 1 and Supplementary Fig. 1)) and the results confirmed undoubtedly the previous association of the MLPH gene locus with this phenotype. We also genotyped a family segregating for the Violet phenotype. This phenotype was chosen based on the fact that farming practices as well as literature indicate that Violet is a triple recessive phenotype clearly involving the Silverblue locus ( $M L P H, p p)$. The family was however non informative for the three markers.

Sequencing of (part of) the genomic region for the MLPH gene in the three coat color phenotypes ( $w t$, Violet and Silverblue) revealed different SNPs, one small insertion between $w t$ and Violet (CATTT in $w t$ changes to GGGGTAGGAA in Violet) at position 5847 and a deletion of the whole intron 7 and part of intron 8 uniquely in Silverblue. Except for the two large deletions for the Silverblue, most of the SNPs and the $5 \mathrm{bp}$ insertion have no functional consequences because they are located in untranslated regions or are synonymous. One not fixed non-synonymous SNP was found in exon 8. Furthermore, an interesting SNP was found at the splice donor site in exon 8/intron 8 of the Violet sequence. In order to investigate the phenomena further, we isolated RNA from $w$, Silverblue and Violet animals and sequenced the $M L P H$ transcript. Surprisingly, Silverblue lacks the entire exon 8 and has also retained part of the intron 8 in the coding region. This introduces a stop codon, leading to a truncated protein 284 residues long that is lacking almost all MYO5A Va and all actin-binding domains. In $w t$ and Violet transcripts, the exon 8 is present but the part of intron 8 is also retained resulting in a protein 359 residues long (lacking the last 28 residues of the MYO5A Va and all actinbinding domains), in comparison to the 572 residues in humans, 590 in mouse and 581 in dog. The retention of part of intron 8 cannot be explained by the mutation in the acceptor splice site in intron 8 because the SNP it is only found in Violet animals.

When comparing the MYO5A Va domain region between the wt mink and the human-mouse-dog there is about $40 \%$ of similarity, in contrast with the RAB27A binding domain, where there is around $95 \%$ of similarity (Fig. 2). When comparing the actin-binding domain between the human, mouse and dog (mink lacks this domain) we can see $73.5 \%$ similarity. These results point towards the fact that the MYO5A Va domain is the lowest conserved domain of the 3 domains present in the MLPH. It is also in this domain that we find a deletion of the last 28 amino acids in mink. Moreover, a 28 amino acid insertion is found in mouse in this domain (Fig. 2). MLPH has been characterized as a linker protein in a tripartite protein complex formed by MYO5A Va and RAB27A. MLPH interacts with RAB27A via its terminal region (amino acids 1-146, light-gray region in Fig. 2) and with MYO5A Va via the central region (amino acids 241 to 405, dark-gray region in Fig. 2). RAB27A encodes a small Ras-like GTPase that seems to play a role in intracellular vesicle transport by controlling vesicle sorting, tethering, docking and fusion, while MYO5A is a protein that moves towards the end of actin filaments and functions in intracellular vesicle transport (i.e. melanosome transport) (Kuroda et al., 2003).

The complex RAB27A-MYO5A Va-MLPH is involved in the capture and short-range actin-based delivery of melanosomes to the melanocytes periphery. This transport is vital to subsequent delivery of the

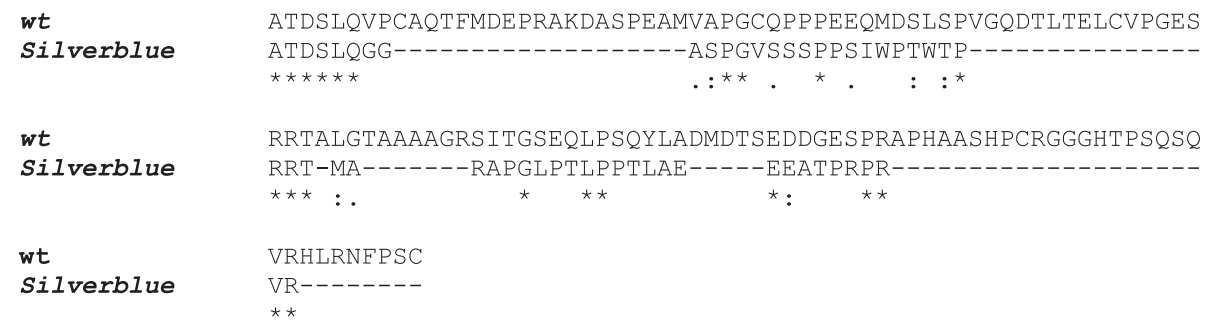

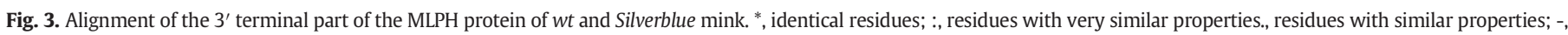
deletion. 


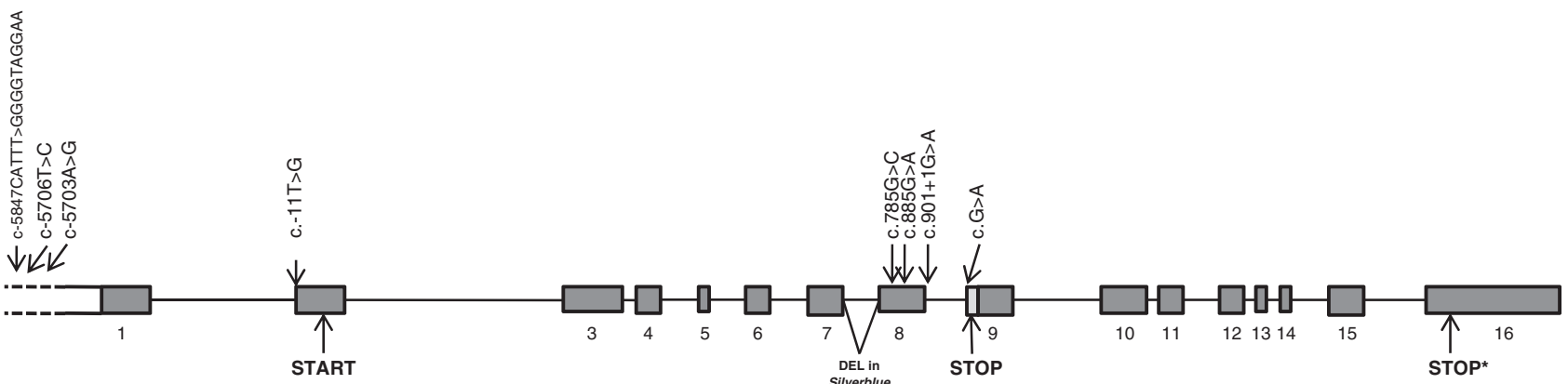

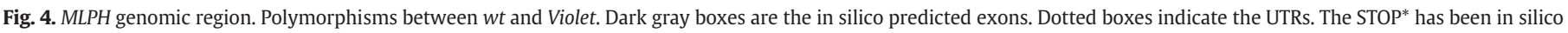

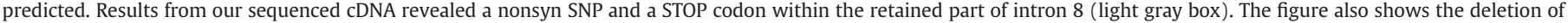
intron 7 in the Silverblue mink.

melanosomes (containing the melanin pigment) into keratinocytes (Fukuda et al., 2002; Kuroda et al., 2003; Nagashima et al., 2002; Provance et al., 2002). A loss of any of the two domains (RAB27A or MYO5A Va), as is the case for the Silverblue phenotype, probably leads to a "recycling" of the melanosomes back to the melanocyte perinuclear area. Thus, the melanophilin with all its three domains is an essential element for proper melanosomes distribution in melanocytes.

Studies in mouse, using deletion mutant (DeltaAB) and point mutants (KA) which lack the actin-binding capacity, have shown that the actin-binding domain is also required for melanosome distribution in melanocytes, together with the RAB27A and MYO5A Va domains (Kuroda et al., 2003). In these studies, the authors conclude that the melanosome transport requires cooperation within the three domains. In contrast, a study of Li et al. (2005) showed that deletion of the actin-binding domain and Rab-binding domain of MLPH did not affect the activation of the ATPase, indicating that the MYO5A Va binding domain is sufficient for the activation of MYO5A Va. The results of our study in mink question the previous investigations by showing that the actin-binding domain seems not to be essential for the transport of melanosomes.

Additionally, transcriptome data derived from 4 different pooled tissues (liver, brain, skin and lung) of the American mink has recently been produced (Anistoroaei et al., 2012a). From this data we could identify both the full length transcript, which is transcribed down to exon 16 (like in other mammals) and a new short transcript (the only one identified in the present study) missing from exon 9 to exon 16, which confirms the findings of this study. Therefore, we cannot dismiss the possibility that the long variant including the actin-binding domain, which was identified in the transcriptome data (Anistoroaei et al., 2012a) still compensates for the actin binding function in other tissues than the ones investigated here (lung and kidney). I.e. the brain tissue included in the transcriptome project was not analyzed in the present study and with its known high gene expression activity it might contain the full length transcript.

In conclusion, wt and Violet are rather similar in the genomic and transcript sequences for the $M L P H$ gene. Contrastingly, the Silverblue phenotype lacks intron 7 and part of intron 8 in the genomic sequence and the complete exon 8 at the cDNA level resulting in a very short and probably non-functional MYO5A Va binding domain. This deficiency would explain the defective distribution of melanosomes in melanocytes culminating in the dilute Silverblue coat color. Furthermore, our study confirms the previously generated information on mink genetics, indicating that more than one allele exists for the Silver locus (Clausen et al., 2012).

The two deletions in the Silverblue MLPH gene and the identified polymorphisms between $w t$ and Violet provide the basis for designing genetic tests to select for Silverblue and Violet carriers respectively in American mink.

Supplementary data to this article can be found online at http:// dx.doi.org/10.1016/j.gene.2013.05.047.

\section{Conflict of interest}

The authors declare no conflicts of interest.

\section{Acknowledgments}

This work was supported by Razvan Anistoroaei's Postdoctoral grant from the Danish Research Council (\#27820) and founding from the Danish Fur Breeders Association. We are thankful to Minna Jakobsen and Anne Strandby for the excellent technical assistance in this project.

\section{References}

Anistoroaei, R., Christensen, K., 2007. Mapping of the silver gene in mink and its association with the dilution gene in dog. Cytogenet. Genome Res. 116, 316-318.

Anistoroaei, R., ten Hallers, B., Nefedov, M., Christensen, K., de Jong, P., 2011. Construction of an American mink Bacterial Artificial Chromosome (BAC) library and sequencing candidate genes important for the fur industry. BMC Genomics 12, 354.

Anistoroaei, R., Krogh, A.K., Christensen, K., 2013. A frameshift mutation in the LYST gene is responsible for the Aleutian colour and the associated Chédiak-Higashi syndrome in American mink. Anim Genet. 44 (2), 178-183.

Anistoroaei, R., Croft, L., Christensen, K., 2012a. An American mink transcriptome. Proceedings of International Society of Animal Genetics; July 15-20, Cairns, Australia, 144, p. P6000.

Anistoroaei, R., Nielsen, V., Markakis, M.N., Karlskov-Mortensen, P., Jørgensen, C.B., Christensen, K., Fredholm, M., 2012b. A re-assigned American mink (Neovison vison) map optimal for genome-wide studies. Gene 511 (1), 66-72.

Barral, D.C., Seabra, M.C., 2004. The melanosome as a model to study organelle motility in mammals. Pigment Cell Res. 17 (2), 111-118 (Apr).

Bed'hom, B., et al., 2008. The lavender plumage colour phenotype in quail is caused by a large deletion in MLPH gene region. Proceedings of the XXXI International Conference of the International Society for Animal Genetics; July, Amsterdam, The Netherlands, pp. 15-20.

Clausen, J., Hansen, B.K., Weiss, V., 2012. In: Copenhagen Fur (Ed.), Mink, bind2 (ISBN 879156672X, 9788791566721)

Drögemüller, C., Philipp, U., Haase, B., Günzel-Apel, A.R., Leeb, T., 2007. A noncoding melanophilin gene (MLPH) SNP at the splice donor of exon 1 represents a candidate causal mutation for coat colour dilution in dogs. J. Hered. 98 (5), 468-473.

Fukuda, M., Kuroda, T.S., Mikoshiba, K., 2002. Slac2-a/melanophilin, the missing link between Rab27 and myosin Va: implications of a tripartite protein complex for melanosome transport. J. Biol. Chem. 277, 12432-12436.

Hume, A.N., Ushakov, D.S., Tarafder, A.K., Ferenczi, M.A., Seabra, M.C., 2007. Rab27a and MyoVa are the primary Mlph interactors regulating melanosome transport in melanocytes. J. Cell Sci. 120, 3111-3122.

Ishida, Y., et al., 2006. A homozygous single-base deletion in MLPH causes the dilute coat colour phenotype in the domestic cat. Genomics 88, 698-705.

Kuroda, T.S., Ariga, H., Fukuda, M., 2003. The actin-binding domain of Slac2-a/melanophilin is required for melanosome distribution in melanocytes. Mol. Cell. Biol. 23, 5245-5255.

Li, X.D., Ikebe, R., Ikebe, M., 2005. Activation of myosin Va function by melanophilin, a specific docking partner of myosin Va. J. Biol. Chem. 280 (18), 17815-17822 (2005 May 6).

Matesic, L.E., et al., 2001. Mutations in Mlph, encoding a member of the Rab effector family, cause the melanosome transport defects observed in leaden mice. Proc. Natl. Acad. Sci. U. S. A. 98, 10238-10243.

Menasche, $G_{\text {. }}$ et al., 2003. Griscelli syndrome restricted to hypopigmentation results from a melanophilin defect (GS3) or a MYO5A F-exon deletion (GS1). J. Clin. Invest. 112 (3), 450-456.

Mercer, J.A., Seperack, P.K., Strobel, M.C., Copeland, N.G., Jenkins, N.A., 1991. Novel myosin heavy chain encoded by murine dilute coat colour locus. Nature 349, 709-713. 
Nagashima, K., et al., 2002. Melanophilin directly links Rab27a and myosin Va through its distinct coiled-coil regions. FEBS Lett. 517, 233-238.

Nascimento, A.A., Roland, J.T., Gelfand, V.I., 2003. Pigment cells: a model for the study of organelle transport. Annu. Rev. Cell Dev. Biol. 19, 469-491.

Passeron, T., Mantoux, F., Ortonne, J.P., 2005. Genetic disorders of pigmentation. Clin. Dermatol. 23 (1), 56-67.

Philipp, U., Hamann, H., Mecklenburg, L., Nishino, S., Mignot, E., Günzel-Apel, A.R., Schmutz, S.M., Leeb, T., 2005. Polymorphisms within the canine MLPH gene are associated with dilute coat colour in dogs. BMC Genet. 6, 34 (Jun 16).
Provance Jr., D.W., James, T.L., Mercer, J.L., 2002. Melanophilin, the product of the leaden locus, is required for targeting of myosin-Va to melanosomes. Traffic 3, 124-132.

Rozen, S., Skaletsky, H., 2000. Primer3 on the WWW for general users and for biologist programmers. Methods Mol. Biol. 132, 365-386.

Vaez, M., Follett, S.A., Bed'hom, B., Gourichon, D., Tixier-Boichard, M., Burke, T., 2008. A single point-mutation within the melanophilin gene causes the lavender plumage colour dilution phenotype in the chicken. BMC Genet. 9, 7 (15).

Wilson, S.M., et al., 2000. A mutation in Rab27a causes the vesicle transport defects observed in ashen mice. Proc. Natl. Acad. Sci. 97, 7933-7938. 